\title{
Catamenial Pneumothorax (CP)
}

\author{
Leonard Ranasinghe ${ }^{1 *}$, Anne Liao ${ }^{2}$ and Michelle Nguyen ${ }^{2}$ \\ ${ }^{1}$ College of Medicine, California Northstate University, USA \\ ${ }^{2}$ Medical Student, California Northstate University, USA
}

*Corresponding author: Leonard Ranasinghe, College of Medicine, California Northstate University, 9700 West Taron Dr. Elk Grove, CA 95757, USA

To Cite This Article: Leonard Ranasinghe. Catamenial Pneumothorax (CP). Am J Biomed Sci \& Res. 2019 - 5(6). AJBSR.MS.ID.000969. DOI: 10.34297/AJBSR.2019.05.000969.

Received: 眥 October 17, 2019; Published: 觜 October 22, 2019

\begin{abstract}
Catamenial pneumothorax (CP) presents as a recurrent respiratory emergency due to failure to identify and treat the underlying gynecological condition. As a rare cause of pneumothorax, CP is unique in its temporal relationship with the onset of menses. Its nature of recurring during time of menstruation makes it less likely to be a separate spontaneous pneumothorax coinciding with menstruation. Several mechanisms have been proposed and although the exact pathophysiology of CP remains elusive, advances in surgical interventions have suggested diaphragmatic defects and thoracic endometriosis to play a fundamental role in the pathogenesis. In addition to technological advances, increased medical awareness and research have suggested that CP may be more common than previously thought. This review article will discuss the proposed mechanism of pathophysiology, evaluation, and acute and long-term management.
\end{abstract}

\section{Introduction}

Catamenial pneumothorax was first described by [1], as a pneumothorax associated with diaphragmatic endometriosis [2]. was the first to coin the term catamenial pneumothorax. Catamenial describes a temporal relationship with the onset of menses, and CP usually presents 1 day to 72 hours within the onset of menses in an ovulating woman. CP is considered a very rare clinical condition. However [3], suggests that CP may be more common than previously suspected in their retrospective review, identifying $8 \mathrm{CP}$ patients out of 32 total pneumothorax female patients $(25 \%)$ referred to their surgical center. According to [4], CP may have been missed before 1990 due to lack of proper visualization of the diaphragm that video-assisted thoracoscopy (VATS) now allows today in pneumothorax patients. Although the exact etiology of CP is unknown, most proposed theories involve the presence of ectopic endometrial tissue in the diaphragm or thorax cavity, known as thoracic endometriosis. CP is the most common presentation of thoracic endometriosis syndrome, over hemothorax, hemoptysis, and parenchymal nodules. In addition, CPs are predominantly right sided (>90\%) as described in current literature. CP should be considered in any woman of child-bearing age with recurrent pneumothoraces. Acute management of $\mathrm{CP}$ should include strong clinical suspicion for a pneumothorax and appropriate placement of chest tube and decompression therapy for the affected lung. Evaluation of a suspected CP includes video- assisted thoracoscopy with a histological confirmation. Prevention of recurrences requires removal of ectopic endometrial tissue and repair of diaphragmatic defects.

\section{Proposed Theories of Etiology}

\section{Prostaglandin theory}

During menstruation, prostaglandins play a critical role in triggering the contraction of uterine muscles to expel menstrual products. Prostaglandin F2 is a particularly potent vasoconstrictor of bronchioles and vascular structures. It is proposed to cause constriction of the bronchioles especially during the expiratory phase, leading to lung collapse and pneumothorax [5]. The research done by Rakhila et al (2015) further supports the prostaglandin theory in the context of endometriosis. They found increased mRNA expression of prostaglandin E2 receptors, prostaglandin F2a receptors, and prostaglandin transporter in ectopic endometrial tissue, increasing cell reactivity and uptake of prostaglandins during menses [6]. This increases the potential for prostaglandins to affect the lungs, although warrants more research.

\section{Coelomic metaplasia}

The pleura, peritoneum, and gonads develop from a common embryologic origin, the coelomic epithelium. Under the influence of estrogen, the pleura and peritoneum has been hypothesized to 
differentiate into endometrial cells, leading to lung damage and pneumothorax [7]. This theory explains endometriosis in patients who have had a hysterectomy or bilateral salpingectomy and in men on high-dose estrogen therapy. However, this theory does not explain why catamenial pneumothoraces are predominantly right sided or intrapulmonary pathologies.

\section{Diaphragmatic theory of air passage}

According to Carter et al (1990) [8], endometrial implants in the pleura and diaphragm creates fenestrations and diaphragmatic pores through cyclical necrosis with menses, allowing air to enter the thorax. Bagan et al (2003)3 identified endometrial tissue around the diaphragmatic performations, which is further evidence of ectopic endometrial tissue to causing diaphragmatic holes. In studies by $[3,5]$, they found underlying diaphragmatic defects in every CP patient, suggesting the fundamental role of the diaphragm in CP. The air that enters the thorax is hypothesized to originate from the fallopian tubes due to a lack of cervical mucus plug during menstruation, then travels through the peritoneum and past the diaphragm. However, this theory does not describe CP in patients that lack fallopian tubes. In addition, air could enter the thorax with every menstrual cycle, but $\mathrm{CP}$ is not observed with every menstrual cycle.

\section{Retrograde menstruation}

This theory is based on the movement of endometrial tissue into the peritoneum, up the right paracolic gutter, and through the diaphragm preferentially on the right side due to the "piston" action of the liver against the diaphragm. The piston effect transmits peritoneal pressure through the diaphragm and allows passage of air and endometrial tissue into thoracic cavity [3]. This explains the occurrence of right sided catamenial pneumothoraces but not left sided. In addition, clockwise peritoneal circulation sweeps endometrial products from the pelvis up the right side of the peritoneal cavity. The falciform ligament halts migration to the left leading to predominantly right sided pneumothoraces.

\section{Clinical Presentation}

Diagnosis of $\mathrm{CP}$ can be challenging but should be suspected in women in their fourth decade of life with recurrent right sided pleuritic chest pain in close temporal relation to onset of menstrual bleeding $[9,10]$. Chest pain the day preceding or within 72 hours of menstrual bleeding is typical of CP. Pain and associated pneumothorax typically occurs on the right side $[9,10]$. Catamenial pneumothorax has late onset, generally in women 30 to 40 years old. A history of pelvic endometriosis may or may not be present, and diaphragmatic defects ranging from 1 to $10 \mathrm{~mm}$ are frequently observed. A subset of $\mathrm{CP}$, termed juvenile catamenial pneumothorax (JCP) has been described in the literature [11]. This is typically earlier in onset, from age 19 and below. JCP has the usual features of close temporality with menses but does not appear to have preferential right sided laterality. A higher frequency of left sided PTX has been observed in this population, and as such has been hypothesized to have a different etiology than usual CP. Furthermore, diaphragmatic defects observed in UCP are generally not seen in JCP.

\section{Evaluation}

Imaging by chest $x$ ray and CT has limited use in diagnosis of CP [10]. Findings on CXR are typically consistent with pneumothorax, generally without mediastinal shift. Findings of pneumoperitoneum may be present10. MRI may show hyperintensities representing endometrial deposits with T1 or T2 weighted images10. CT may also be useful in imaging endometrial lesions [10]. However, as previously discussed, endometrial deposits may not be present in cases of $\mathrm{CP}$. Therefore, the gold standard in diagnosis of $\mathrm{CP}$ remains video-assisted thoracoscopy (VATS) [10] in combination with histologic confirmation. Diaphragmatic defects and other lesions within the thoracic cavity may be easily visualized and repaired with VATS.

\section{Management \& Complications}

For acute management of pneumothorax, appropriate treatment with placement of a chest tube or decompression to reinflate the lung may be indicated [12]. Historically, axillary thoracotomy was the mainstay for treatment of CP [13]. However, axillary thoracotomy presented several difficulties, particularly in that visualization of the diaphragm was virtually impossible. VATS provides an effective means of visualizing the diaphragm and thoracic cavity, and allows good access to repair any perforations or other defects observed13. The literature describes several different methods of repair, including removal of defects by stapler, simple suturing of defects, and plication $[5,13,14]$. A retrospective study by Bagan et al advocates for the use of the application of glue in combination with polyglactin mesh to the diaphragm to treat perforations which may not be visualized, and promote fibrotic adhesion of the diaphragm with the lung to prevent recurrence4. Repair of defects is typically followed by pleurectomy or pleurodesis by mechanical or chemical means (Talc powder) to promote fibrosis and adhesion between lung and diaphragmatic surfaces3. Surgical intervention is followed with medical treatment to prevent recurrence [15].

Medical treatment for CP aims to suppress ovulation [10], and options for treatment are similar to those used in management of endometriosis. These include gonadotropin releasing hormone (GnRH) analogs, danazol, oral contraceptive agents, and progestin drugs. Pregnancy, and consequent anovulation, has also been observed to suppress episodes of CP [9]. Use of GnRH analogs is typically limited to 6 to 12 months due to side effects 14 of hypoestrogenism, particularly decreased trabecular bone density, vaginal dryness, and hot flashes. Bone density is typically restored after 2 years of GnRH analog cessation. Recurrence of CP has been observed in some cases with delayed initiation of medical treatment following surgical intervention [14], but there is some evidence in 
the literature that appropriate surgical treatment alone may be effective in prevention of recurrence [16].

\section{Acknowledgements}

We would like to thank Dr. Leonard Ranasinghe for his assistance and mentorship.

\section{References}

1. Maurer ER, Schaal JA, Mendez FL (1958) Chronic recurring spontaneous pneumothorax due to endometriosis of the diaphragm. Journal of the American Medical Association 168(15): 2013-2014.

2. Lillington GA, Mitchell SP, Wood GA (1972) Catamenial pneumothorax Jama 219(10): 1328-1332.

3. Alifano M, Roth T, Broet SC, Schussler O, Magdeleinat P, et al. (2003) Catamenial pneumothorax: a prospective study. Chest 124(3): 10041008.

4. Bagan P, Barthes FLP, Assouad J, Souilamas R, Riquet M (2003) Catamenial pneumothorax: retrospective study of surgical treatment. The Annals of thoracic surgery 75(2): 378-381.

5. Leong AC, CoonarAS, Lang Lazdunski L(2006) Catamenial pneumothorax: surgical repair of the diaphragm and hormone treatment. The Annals of The Royal College of Surgeons of England 88(6): 547-549.

6. Rakhila H, Bourcier N, Akoum A, Pouliot, M. (2015). Abnormal expression of prostaglandins E2 and $\mathrm{F} 2 \alpha$ receptors and transporters in patients with endometriosis. BioMed research international 2015: 808146.

7. Matsuura K, Ohtake H, Katabuchi H, Okamura H (1999) Coelomic metaplasia theory of endometriosis: evidence from in vivo studies and an in vitro experimental model. Gynecologic and obstetric investigation 47(Suppl 1): 18-22.

8. Carter EJ, Ettensohn DB (1990) Catamenial pneumothorax. Chest 98(3): 713-717.

9. Rossi NP, Goplerud CP (1974) Recurrent catamenial pneumothorax. Archives of Surgery 109(2): 173-176.

10. Narula N, Ngu S, Avula A, Mansour W, Chalhoub M (2018) Left-sided Catamenial Pneumothorax: A Rare Clinical Entity. Cureus 10(5): e2567.

11. Inoue T, Chida M, Inaba H, Tamura M, Kobayashi S, et al. (2015) Juvenile catamenial pneumothorax: institutional report and review. Journal of cardiothoracic surgery 10(1): 83.

12. Arunthari V, Sevin BU, Krishna M, Johnson MM (2008) Catamenial pneumothorax with umbilical and diaphragmatic endometriosis: a case report and review of the literature. Southern medical journal 101(10): 1043-1045.

13. Hazelrigg SR (2003) Secondary spontaneous pneumothorax: catamenial pneumothorax. Chest 124(3): 781-782.

14. Attaran S, Bille A, Karenovics W, Lang Lazdunski L (2013) Videothoracoscopic repair of diaphragm and pleurectomy/abrasion in patients with catamenial pneumothorax: a 9-year experience. Chest 143(4): 1066-1069.

15. Black MC, Joubert K, Seese L, Ocak I, Frazier A, et al. (2019) Innovative and Contemporary Interventions of Diaphragmatic Disorders. Journal of thoracic imaging 34(4): 236-247.

16. Subotic D, Mikovic Z, Atanasijadis N, Savic M, Moskovljevic D (2016) Hormonal therapy after the operation for catamenial pneumothorax-is it always necessary? Journal of cardiothoracic surgery 11(1): 66. 